\title{
1 Simple method for measuring center of mass work during field running
}

2

3 Authors:

4 Marine Devise ${ }^{1,2}$, Jérémy Rossi ${ }^{1}$, Nicolas Theveniau ${ }^{2}$, Alain Belli ${ }^{1}$

5

\section{Affiliation:}

$7{ }^{1}$ Univ Lyon, UJM-Saint-Etienne, Laboratoire Interuniversitaire de Biologie de la Motricité, 8 EA 7424, F-42023 Saint-Etienne, France

$9 \quad{ }^{2}$ CTC, Comité Professionnel de Développement Cuir Chaussure Maroquinerie, 4 rue Hermann 10

Corresponding author: Marine Devise

Address: Campus Santé Innovation - 10 Rue de la Marandière - 42270 SAINT-PRIEST-ENJAREZ - FRANCE

Tel: +33477481500

Email: marine.devise@univ-st-etienne.fr

Submission for publication: Short communication

\section{Word count (Introduction through Discussion): 2094}

20

Keywords: total center of mass work, leg stiffness, running 


\section{Abstract}

24 The purpose of this study was to propose and validate a new simple method for calculation of center of mass work during field running, in order to avoid the use of costly and inconvenient measurement devices. This method relies on spring-mass model and measurements of average horizontal velocity, and contact and flight times during running. Ten male, recreational subjects ran on a dynamometer treadmill at different velocities ranging from 2.22 to $4.44 \mathrm{~m} . \mathrm{s}^{-1}$ during 4 $\min 30 \mathrm{~s}$ for each velocity. Twenty consecutive steps were analyzed after 3 min $30 \mathrm{~s}$. The potential $\left(W_{\text {pot }}\right)$, forward kinetic $\left(W_{\text {kinf }}\right)$ and the total center of mass $\left(W_{\text {ext }}\right)$ work data obtained with this new method were compared with the reference data calculated from ground reaction force measurements. $W_{\text {ext }}, W_{\text {pot }}$ and $W_{\text {kinf }}$ values calculated with the proposed method were respectively $+3.39 \pm 0.77 \%$ higher, $-4.14 \pm 0.72 \%$ lower and $+7.34 \pm 1.08 \%$ higher than values obtained by the reference method. Furthermore, significant linear regressions close to the identity line were obtained between the reference and the proposed method values of works ( $\mathrm{r}$ $=0.99, \mathrm{p}<0.05$ for $W_{\text {ext }} ; \mathrm{r}=0.98, \mathrm{p}<0.05$ for $W_{\text {pot }} ; \mathrm{r}=0.98, \mathrm{p}<0.05$ for $\left.W_{\text {kinf }}\right)$. It was concluded that this new method could provide a good estimate of center of mass work in field running thanks to a few simple mechanical parameters. 


\section{Introduction}

In sports, the measurement of mechanical power and/or work is often used for research or athlete training and monitoring purposes. As recently mentioned by van der Kruk et al. (2018): "The quantification of mechanical power can provide valuable insight into athlete performance because it is the mechanical principle of the rate at which the athlete does work or transfers energy to complete a movement task".

The biomechanics and energetics of running have been mainly investigated by means of two integrative mechanical models: the "center of mass model" (CMM) and the "spring-mass model" (SMM). The work done to raise and accelerate the center of mass (COM) at each step was defined as "external work" ( $W_{\text {ext }}$, Cavagna, 1975; Fenn, 1930) or center of mass work (van der Kruk et al., 2018) in the literature. The SMM is widely used for describing the stored and released elastic energy in the lower limbs in humans at each step. The SMM is mainly characterized and controlled by the stiffness of the leg $\left(k_{\text {leg }}\right)$ during contact (McMahon and Cheng, 1990; Farley and González, 1996) in the sense that the SMM represents the bounce of the body on the ground by a single mass mounted on a springy leg.

CMM and SMM biomechanical models have been used in the literature to address the differences in metabolic energy cost among individuals. Indeed, inter-individual variability of the energy cost of running $\left(\mathrm{C}_{\mathrm{r}}\right)$ have been significantly related to mechanical parameters and more specifically to $W_{\text {ext }}$ (Saibene and Minetti, 2003) and $k_{\text {leg }}$ (Dalleau et al., 1998). Significant positive correlations between inter- and intra-individual $\mathrm{C}_{\mathrm{r}}$ and $W_{\text {ext }}$ variations were found by Bourdin et al. (1995) for an homogeneous group of long distance runners and by Lazzer et al. (2014) in ultra-marathon runners. Furthermore, $k_{l e g}$ was negatively correlated with inter-individual variability of $\mathrm{C}_{\mathrm{r}}$ for middle-distance runners (Dalleau et al., 1998).

Therefore, the measurements of $W_{\text {ext }}$ and $k_{\text {leg }}$ are important to further investigate the biomechanics of running. In laboratory conditions, $W_{\text {ext }}$ can be accurately measured (Cavagna, 1975; Arampatzis et al., 2000; Saibene and Minetti, 2003; Avogadro et al., 2004; Pavei et al., 2017) by using force platforms or instrumented treadmills and motion capture systems. Nowadays, these techniques are still used in order to find any small differences that could discriminate variations among subjects. Using the same measurement devices, $k_{\text {leg }}$ was also initially investigated in laboratory conditions (McMahon and Cheng, 1990; Farley and González, 1996). In 2005, Morin et al. proposed and validated a simple method for measurements of $k_{\text {leg }}$, based on measurement of body mass and leg length and on field 
measurement of contact time, flight time and average running speed. The contact and flight times as well as average running speed can nowadays be performed by portable and lightweight equipment such as pressure sensors, accelerometers, goniometers or gyroscopes (Norris et al., 2014), or by systems utilizing photocell beams placed on running tracks (Glatthorn et al., 2011). The Morin's method has since been widely used in field conditions (Hobara et al. 2010; Morin et al. 2011a, b).

To the best of our knowledge, there are no simple methods that could monitor $W_{\text {ext }}$ in field running conditions and the purpose of the present study was to propose and validate such a method. The proposed method is based on the same measurements as Morin et al. (2005): contact time, flight time, average running speed, leg length and body mass. It was validated during treadmill running by comparing it with the reference $W_{\text {ext }}$ computed from ground reaction forces (GRF, Avogadro et al., 2004; Cavagna, 1975).

\section{Materials and methods}

\section{Protocol}

Ten male subjects ( $22.5 \pm 2.84$ years; $75.6 \pm 6.05 \mathrm{~kg} ; 1.82 \pm 0.06 \mathrm{~m}$; leg length $0.96 \pm 0.04 \mathrm{~m}$; mean $\pm \mathrm{SD}$ ) gave their informed consent to participate in this study. They were all physical education students and experienced in treadmill running. After a standardized 6 min warm-up at $3.33 \mathrm{~m} . \mathrm{s}^{-1}$, subjects ran $4 \mathrm{~min} 30 \mathrm{~s}$ on a treadmill at $2.22,2.78,3.33,3.89$ and $4.44 \mathrm{~m} . \mathrm{s}^{-1}$, in random order, interspersed with at least 5 min rest.

Mechanical parameters were measured by treadmill dynamometer (ADAL 3DC, HEF Techmachine, Andrézieux-Bouthéon, France, for details, see Belli et al. (2001)). Force data were collected after $3 \min 30 \mathrm{~s}$. 3D GRF were sampled at $1000 \mathrm{~Hz}$ and were filtered using a low-pass fourth-order Butterworth filter with a $50 \mathrm{~Hz}$ cut-off. Contact and aerial times were measured from force signals, contact period corresponded to a vertical force higher than $30 \mathrm{~N}$. Measurements were calculated for each cycle (defined by time period from one heel strike to contralateral heel strike) then averaged for twenty consecutive steps to obtain a good estimate of average step values (Belli et al., 1995).

Reference method for measuring $W_{\text {ext }}$ 
The reference forward kinetic work $\left(W_{\text {kinf }}\right)$ due to forward acceleration of the body and the reference potential work $\left(W_{p o t}\right)$ done to raise the COM were calculated from GRF of the foot during each step and computed the speeds and displacement of the COM by simple timeintegration of antero-posterior GRF and double time-integration of vertical GRF respectively, according to the method of Avogadro et al. (2004). Assuming that no transfer occurred between kinetic and potential energies in running (Cavagna et al., 1964) and that the medio-lateral work was negligible (Cavagna, 1975), $W_{\text {ext }}$ was calculated as:

$W_{\text {ext }}=W_{\text {kinf }}+W_{\text {pot }}$

\section{Proposed method for measuring $\widehat{W}_{\text {ext }}$}

In the present method the $\widehat{W}_{\text {ext }}$ was simply computed as the elastic mechanical energy stored and released by the spring-mass model (e.g. Blickhan and Full, 1993):

$\widehat{W}_{\text {ext }}=\frac{1}{2} k_{\text {leg }} \Delta L^{2}$

where $k_{\text {leg }}$ (in $\mathrm{kN} \cdot \mathrm{m}^{-1}$ ) and $\Delta L$ (in m) were calculated according to the method of Morin et al. (2005):

$k_{\text {leg }}=F_{\text {max }} \Delta L^{-1}(3) \quad$ and $\quad \Delta L=L-\sqrt{L^{2}-\left(\frac{v t_{c}}{2}\right)^{2}}+\Delta y(4)$

with $\quad F_{\max }=m g \frac{\pi}{2}\left(\frac{t_{f}}{t_{c}}+1\right)(5) \quad$ and $\quad \Delta y=\left|-\frac{F_{\max } t_{c}^{2}}{m \pi^{2}}+g \frac{t_{c}{ }^{2}}{8}\right|$

Where $m$ is the body mass (in $\mathrm{kg}$ ), $g=9.81 \mathrm{~m} . \mathrm{s}^{-2}, v$ the average running velocity (in $\mathrm{m} . \mathrm{s}^{-1}$ ), $L$ the leg length (in $\mathrm{m}$ ), $t_{c}$ and $t_{f}$, the contact and the flight times (in s) respectively determined from vertical GRF.

However, in former $\Delta L$ computations (equation 4) it is assumed that the average horizontal running velocity during the contact phase $\left(v_{c o n}\right)$ is the same than the average running velocity (v) (e.g. Farley et al. 1993). Because the horizontal velocity is maximal in flight phase and lower in contact phase (Blickhan, 1989; Cavagna, 2006), $\Delta L$ was computed using $v_{c o n}$ in the present study (see appendix):

$\Delta L^{\prime}=L-\sqrt{L^{2}-\left(\frac{v_{c o n} t_{c}}{2}\right)^{2}}+\Delta y$ 
Furthermore, variations of trunk inclination in sagittal plane during the contact phase of running

129 (Thorstensson et al., 1984) induce a backward movement of the COM of the trunk. Therefore, the real horizontal displacement of the COM is lower than the horizontal displacement $v_{c o n} t_{c}$ calculated with the SMM (equation 7) that assumes that the COM is always located at the upper end of the leg. This backward movement increases with velocity starting from $2 \mathrm{~cm}$ at $2 \mathrm{~m} . \mathrm{s}^{-1}$ to $3 \mathrm{~cm}$ at $5 \mathrm{~m} . \mathrm{s}^{-1}$ at L3 vertebral level (Thorstensson et al., 1984, figure 5) and could induce an increasing overestimation of $\Delta L$ with velocity. Taking this phenomenon into account, $\Delta L$ was recalculated:

$\Delta L^{\prime \prime}=L-\sqrt{L^{2}-\left(\frac{v_{c o n} t_{c}-0,02-\frac{\left(v_{c o n}-2\right)}{3} \times(0.03-0.02)}{2}\right)^{2}}+\Delta y$

From only $t_{c}$ and $t_{f}$ measurements $\widehat{W}_{p o t}$ could also be calculated:

$\widehat{W}_{p o t}=m g\left(\Delta y+\frac{1}{8} g t_{f}^{2}\right)$

where $\frac{1}{8} g t_{f}^{2}$ correspond to the rising of the COM during the flight time (Bosco et al., 1983).

It was then possible to obtain $\widehat{W}_{\text {kinf }}$ according to equation (1):

$\widehat{W}_{\text {kinf }}=\widehat{W}_{\text {ext }}-\widehat{W}_{\text {pot }}(10)$

\section{Statistical analysis}

The standard errors of estimate (SEE) of main parameters $\left(W_{\text {ext }}, W_{\text {kinf }}\right.$ and $\left.W_{\text {pot }}\right)$ were computed for each subject between values obtained from force plate data at each step and values estimated from model computations:

$S E E=\sqrt{\frac{\sum\left(F_{\text {Reference }}-F_{\text {Model }}\right)^{2}}{N_{\text {steps }}-2}}$

Absolute bias was calculated for each subject at each velocity:

absolute bias $=\left|\frac{\text { Model-Reference }}{\text { Reference }}\right| \times 100$ (12)

The reference-model relationships were further described by the calculation of the Pearson's correlation coefficient (r). To analyze the reliability of the proposed method in comparison with the reference, the Intraclass correlation coefficient was used (ICC-2,1). Statistical significance 
was accepted at $\mathrm{p}<0.05$. All data processing was performed using Matlab 2016b (The MathWorks Inc., Natick, MA).

\section{Results}

The values obtained with both the reference and proposed method, absolute reference-proposed bias, Pearson's correlation coefficient and SEE are shown in Table 1. The proposed method overestimated $\widehat{W}_{\text {ext }}$ and $\widehat{W}_{\text {kinf }}$, and underestimated $\widehat{W}_{\text {pot }}$ as compared with the reference method. $\widehat{W}_{\text {ext }}$ mean bias was $3.39 \%$, ranging from 2.64 to $4.36 \%$. $\widehat{W}_{\text {pot }}$ and $\widehat{W}_{\text {kinf }}$ biases were respectively $4.14 \%$ (ranging from 2.07 to $7.84 \%$ ) and $7.34 \%$ (ranging from 6.70 to $8.12 \%$ ).

There were excellent agreements between methods for $W_{\text {ext }}, W_{\text {pot }}$ and $W_{\text {kinf }}(\mathrm{ICC}=0.976, \mathrm{p}$ $<0.001 ;$ ICC $=0.952, \mathrm{p}<0.01$ and ICC $=0.960, \mathrm{p}<0.001$ respectively). The values of $\widehat{W}_{\text {ext }}$, $\widehat{W}_{\text {pot }}$ and $\widehat{W}_{\text {kinf }}$ were very close to the reference values measured at each step, with SEE of

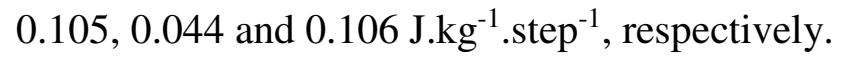

The reference-model linear regressions of mechanical work (figure 1) were highly significant and Pearson's correlation coefficient revealed a very high relationship for $W_{\text {ext }}, W_{\text {pot }}$ and $W_{\text {kinf }}(\mathrm{r}=0.99, \mathrm{p}<0.001 ; \mathrm{r}=0.98, \mathrm{p}<0.001 ; \mathrm{r}=0.98, \mathrm{p}<0.001$ respectively). Nevertheless, there seems to be a tendency of under/over-estimation of the proposed Wext method with respectively lower/higher running speeds.

$$
\begin{aligned}
& * * * * * \mathrm{TABLE} 1 * * * * * * \\
& * * * * * \mathrm{FIGURE} 1 * * * * *
\end{aligned}
$$

\section{Discussion}

The purpose of this study was to provide a calculation method of $W_{\text {ext }}$ based on simple measurements. When this calculation method was compared to reference measurements a high level of agreement of $\widehat{W}_{\text {ext }}$ (showed by high ICC) and a very high coefficient correlation $(\mathrm{r}=0.99, \mathrm{p}<0.01)$ were observed. The same conclusions were reached for $\widehat{W}_{\text {pot }}$ and $\widehat{W}_{k i n f}$. Furthermore, the mean biases of the present study (3.39\% for $W_{\text {ext }}, 4.14 \%$ for $W_{\text {pot }}$ and $7.34 \%$ for $W_{\text {kinf }}$ ) were comparable to Morin et al. (2005) with bias of $6.05 \%$ for $k_{\text {leg }}$ during treadmill running (between 3.33 and $6.67 \mathrm{~m} \cdot \mathrm{s}^{-1}$ ). These biases could be explained by the limits inherent 
to the spring-mass model, i.e., the human lower limb is not a true linear spring in a physical sense. This point has been widely discussed in the literature (Blickhan, 1989; McMahon and Cheng, 1990; Latash and Zatsiorsky, 1993; Farley and González, 1996).

The values of $W_{\text {ext }}, W_{\text {pot }}$ and $W_{\text {kinf }}$ obtained in the present study are in agreement with the range of values reported in the literature for comparable running velocities (Arampatzis et al., 2000; Avogadro et al., 2004; Bourdin et al., 1995; Cavagna et al., 1964; Dalleau et al., 1998;

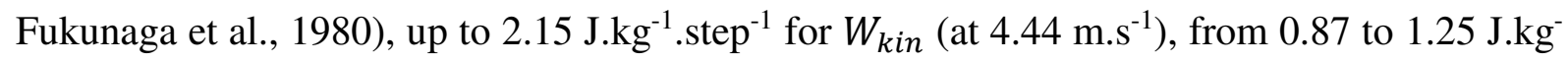

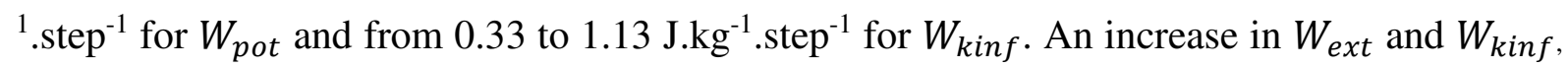
and a constant $W_{\text {pot }}$ with velocity were also reported in the literature (Cavagna et al., 1964; Avogadro et al., 2004).

It is also important to note that the present method is based on a single body model and thus limited to the calculations of work done by the COM. Minetti (1998) already proposed a method to estimate the "internal work" $\left(W_{\text {int }}\right)$ produced during field running. This method requires the same type of measurements: running speed, contact and flight times. Both methods could then be combined to obtain both $W_{\text {ext }}$ and $W_{\text {int }}$ in field conditions. As discussed by van der Kruk et al. (2018) in a recent review, the definition of $W_{\text {int }}$ is not consistent. These authors also mentioned that multi-joints models and inverse dynamics could be used to calculate and sum joint powers. However, because there is no standard on whether to allow for energy flow between joints, large discrepancy on total work and power calculations can be found among papers using multi-joint models. In addition, in field conditions the frictional work and power mainly due to air friction cannot be taken into account by either single joint nor multi-joint models, but could be estimated from running velocity (Davies, 1980). The energy cost of overcoming air resistance on a calm day outdoor was calculated to be $2 \%$ in marathon $\left(5 \mathrm{~m} . \mathrm{s}^{-}\right.$ ${ }^{1}$ ) up to $7.8 \%$ for sprinting $\left(10 \mathrm{~m} \cdot \mathrm{s}^{-1}\right)$. Within the velocity range of the present study it represents less than $2 \%$ of the total $\mathrm{C}_{\mathrm{r}}$.

Although the present study provides a new mathematical methodology of mechanical work, its extrapolation on overground conditions must be interpreted with caution, since it has not been field tested. Although no fundamental differences exist between mechanics of overground versus treadmill locomotion (van Ingen Schenau, 1980), the wearables used may not be as accurate as the instrumented treadmill to measure horizontal velocity, $t_{f}$ and $t_{c}$. Further investigations are then needed to fully validate this method in field condition. 
213 To sum up, $\widehat{W}_{\text {ext }}$ appears to provide consistent calculations of mechanical work production

214 during steps. In future, the present method could be used to monitor $\widehat{W}_{\text {ext }}$ changes during long distance field running and with shoes or tracks having different damping and elastic characteristics. Coupled with lightweight and portable tool, such a method could provide mechanical feedback on running techniques during normal trainings and/or competitions.

To our knowledge, this is the first method that could be applied to $W_{\text {ext }}$ in field conditions. This new calculation is both a valid and reliable method to estimate $W_{\text {ext }}$ thanks to simple parameters and practical for field running.

\section{Acknowledgements}

The authors would like to thank the subjects of the present study for their participation and Camille Perrin and Florian Rifino for their contribution during the experiments.

\section{Conflict of Interest}

The authors do not have any conflict of interest or personal relationships with other people or organizations that could inappropriately influence this work.

\section{References}

Arampatzis, A., Knicker, A., Metzler, V., Brüggemann, G.P., 2000. Mechanical power in running: a comparison of different approaches. Journal of Biomechanics. 33, 457-463.

Avogadro, P., Chaux, C., Bourdin, M., Dalleau, G., Belli, A., 2004. The use of treadmill ergometers for extensive calculation of external work and leg stiffness during running. European Journal of Applied Physiology. 92, 182-185.

Belli, A., Bui, P., Berger, A., Geyssant, A., Lacour, J.R., 2001. A treadmill ergometer for threedimensional ground reaction forces measurement during walking. Journal of Biomechanics. $34,105-112$.

Belli, A., Lacour, J.R., Komi, P.V., Candau, R., Denis, C., 1995. Mechanical step variability during treadmill running. European Journal of Applied Physiology and Occupational Physiology. 70, $510-517$.

Blickhan, R., 1989. The spring-mass model for running and hopping. Journal of Biomechanics. 22, $1217-1227$. 
Blickhan, R., Full, R.J., 1993. Similarity in multilegged locomotion: Bouncing like a monopode. Journal of Comparative Physiology A. 173, 509-517.

Bosco, C., Luhtanen, P., Komi, P.V., 1983. A simple method for measurement of mechanical power in jumping. European Journal of Applied Physiology and Occupational Physiology. 50, 273-282.

Bourdin, M., Belli, A., Arsac, L.M., Bosco, C., Lacour, J.R., 1995. Effect of vertical loading on energy cost and kinematics of running in trained male subjects. Journal of Applied Physiology. 79, 2078-2085.

Cavagna, G.A., 1975. Force platforms as ergometers. Journal of Applied Physiology. 39, 174-179.

Cavagna, G.A., 2006. The landing-take-off asymmetry in human running. Journal of Experimental Biology. 209, 4051-4060.

Cavagna, G.A., Saibene, F.P., Margaria, R., 1964. Mechanical work in running. Journal of Applied Physiology. 19, 249-256.

Dalleau, G., Belli, A., Bourdin, M., Lacour, J.R., 1998. The spring-mass model and the energy cost of treadmill running. European Journal of Applied Physiology. 77, 257-263.

Davies, C.T., 1980. Effects of wind assistance and resistance on the forward motion of a runner. Journal of Applied Physiology. 48, 702-709.

Farley, C.T., Glasheen, J., McMahon, T.A., 1993. Running springs: speed and animal size. Journal of Experimental Biology. 185, 71-86.

Farley, C.T., González, O., 1996. Leg stiffness and stride frequency in human running. Journal of Biomechanics. 29, 181-186.

Fenn, W.O., 1930. Work against gravity and work due to velocity changes in running. American Journal of Physiology-Legacy Content. 93, 433-462.

Fukunaga, T., Matsuo, A., Yuasa, K., Fujimatsu, H., Asahina, K., 1980. Effect of running velocity on external mechanical power output. Ergonomics. 23, 123-136.

Glatthorn, J.F., Gouge, S., Nussbaumer, S., Stauffacher, S., Impellizzeri, F.M., Maffiuletti, N.A., 2011. Validity and reliability of Optojump photoelectric cells for estimating vertical jump height. Journal of Strength and Conditioning Research. 25, 556-560.

Hobara, H., Inoue, K., Gomi, K., Sakamoto, M., Muraoka, T., Iso, S., Kanosue, K., 2010. Continuous change in spring-mass characteristics during a 400m sprint. Journal of Science and Medicine in Sport. 13, 256-261.

Latash, M.L., Zatsiorsky, V.M., 1993. Joint stiffness: Myth or reality? Human Movement Science. 12, 653-692.

Lazzer, S., Taboga, P., Salvadego, D., Rejc, E., Simunic, B., Narici, M.V., Buglione, A., Giovanelli, N., Antonutto, G., Grassi, B., Pisot, R., di Prampero, P.E., 2014. Factors affecting metabolic cost of transport during a multi-stage running race. Journal of Experimental Biology. 217, 787-795. 
McMahon, T.A., Cheng, G.C., 1990. The mechanics of running: How does stiffness couple with speed? Journal of Biomechanics. 23, 65-78.

Minetti, A.E., 1998. A model equation for the prediction of mechanical internal work of terrestrial locomotion. Journal of Biomechanics. 31, 463-468.

Morin, J.B., Dalleau, G., Kyröläinen, H., Jeannin, T., Belli, A., 2005. A simple method for measuring stiffness during running. Journal of Applied Biomechanics. 21, 167-180.

Morin, J.B., Samozino, P., Millet, G.Y., 2011a. Changes in running kinematics, kinetics, and springmass behavior over a 24-h run. Medicine \& Science in Sports \& Exercise. 43, 829-836.

Morin, J.B., Tomazin, K., Edouard, P., Millet, G.Y., 2011b. Changes in running mechanics and spring-mass behavior induced by a mountain ultra-marathon race. Journal of Biomechanics. 44, 1104-1107.

Norris, M., Anderson, R., Kenny, I.C., 2014. Method analysis of accelerometers and gyroscopes in running gait: A systematic review. Proceedings of the Institution of Mechanical Engineers, Part P: Journal of Sports Engineering and Technology. 228, 3-15.

Pavei, G., Seminati, E., Storniolo, J.L.L., Peyré-Tartaruga, L.A., 2017. Estimates of Running Ground Reaction Force Parameters from Motion Analysis. Journal of Applied Biomechanics. 33, 6975.

Saibene, F., Minetti, A.E., 2003. Biomechanical and physiological aspects of legged locomotion in humans. European Journal of Applied Physiology. 88, 297-316.

Thorstensson, A., Nilsson, J., Carlson, H., Zomlefer, M.R., 1984. Trunk movements in human locomotion. Acta Physiologica Scandinavica. 121, 9-22.

van der Kruk, E., van der Helm, F.C.T., Veeger, H.E.J., Schwab, A.L., 2018. Power in sports: A literature review on the application, assumptions, and terminology of mechanical power in sport research. Journal of Biomechanics. 79, 1-14.

van Ingen Schenau, G.J., 1980. Some fundamental aspects of the biomechanics of overground versus treadmill locomotion. Medicine and Science in Sports and Exercise. 12, 257-261. 


\section{APPENDIX}

Computation of average velocity during contact time:

309 The average running velocity during a complete step $(v)$ is given by:

$$
v=\frac{v_{f} \times t_{f}+v_{\text {con }} \times t_{c}}{t_{c}+t_{f}}=\frac{v_{\max } \times t_{f}+\left(v_{\max }-\frac{\Delta v}{2}\right) \times t_{c}}{t_{c}+t_{f}}=v_{\max }-\frac{\Delta v \times t_{c}}{2 \times\left(t_{c}+t_{f}\right)}
$$

311 where $v_{f}=v_{\max }$ is the maximal velocity during the flight phase, $v_{c o n}$ is the average velocity

312 during contact and $\Delta v$ is the change of velocity due to deceleration and corresponding 313 acceleration during contact phase.

314 It is then possible to establish a relationship between the two unknowns $v_{\max }$ and $\Delta v$ :

$$
v_{\max }=v+\frac{\Delta v \times t_{c}}{2 \times\left(t_{c}+t_{f}\right)}
$$

316 A second relationship between $v_{\max }$ and $\Delta v$ can be drawn from $\widehat{W}_{k i n f}$ as follow:

$\widehat{W}_{k i n f}=\frac{1}{2} m\left(v_{\max }^{2}-v_{\min }^{2}\right)=\frac{1}{2} m\left(v_{\max }^{2}-\left(v_{\max }-\Delta v\right)^{2}\right)$

Combining equations ( $\left(1^{\prime}\right)$ and (2') we get a second degree equation of $\Delta v$ :

$$
\frac{t_{f}}{t_{c}+t_{f}} \Delta v^{2}+(-2 v \Delta v)+\frac{2 \widehat{W}_{k i n f}}{m}=0
$$

321 with two $\Delta v$ real solutions, using the only solution that gives $\Delta v<v$ it is finally possible to

322 compute $v_{c o n}$ from equation (1):

$$
v_{\text {con }}=v_{\max }-\frac{\Delta v}{2} \times \frac{t_{f}}{t_{c}+t_{f}}
$$




\section{Figure 1}

(A)

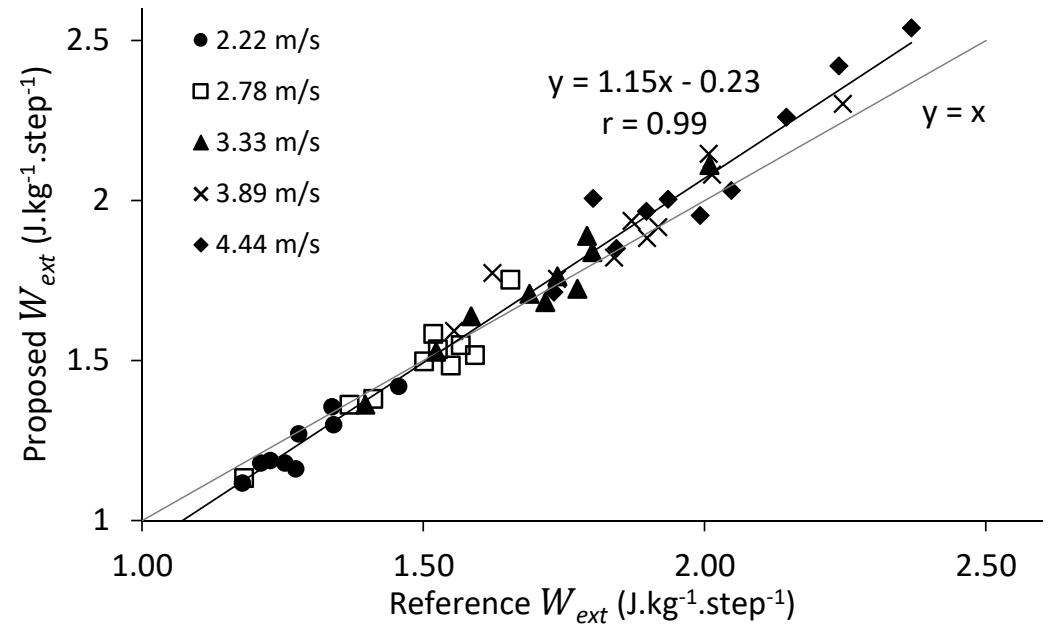

(B)

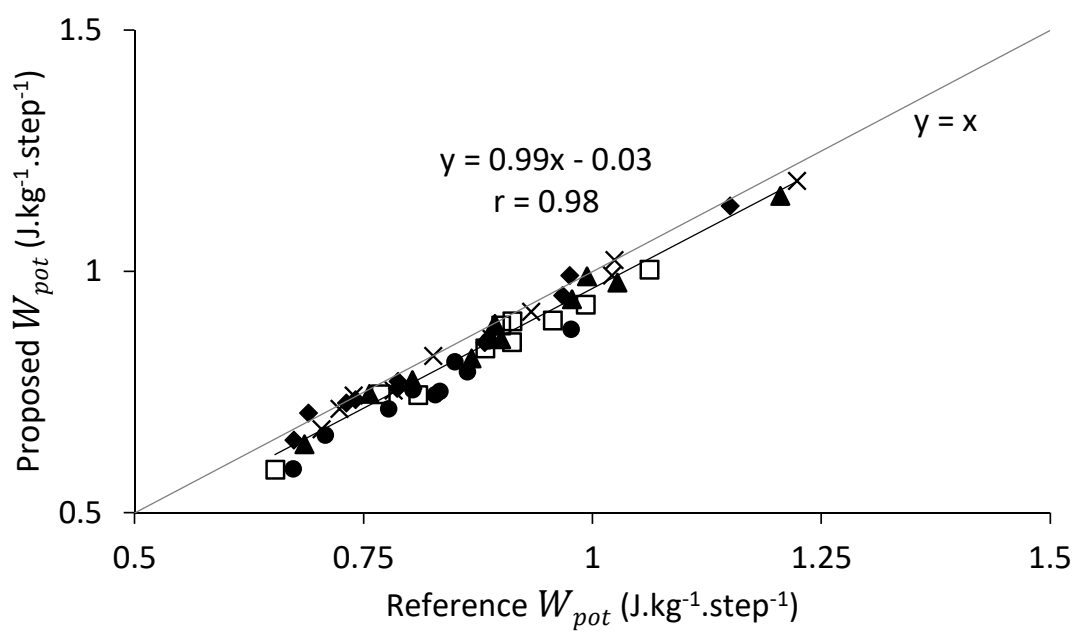

(C)

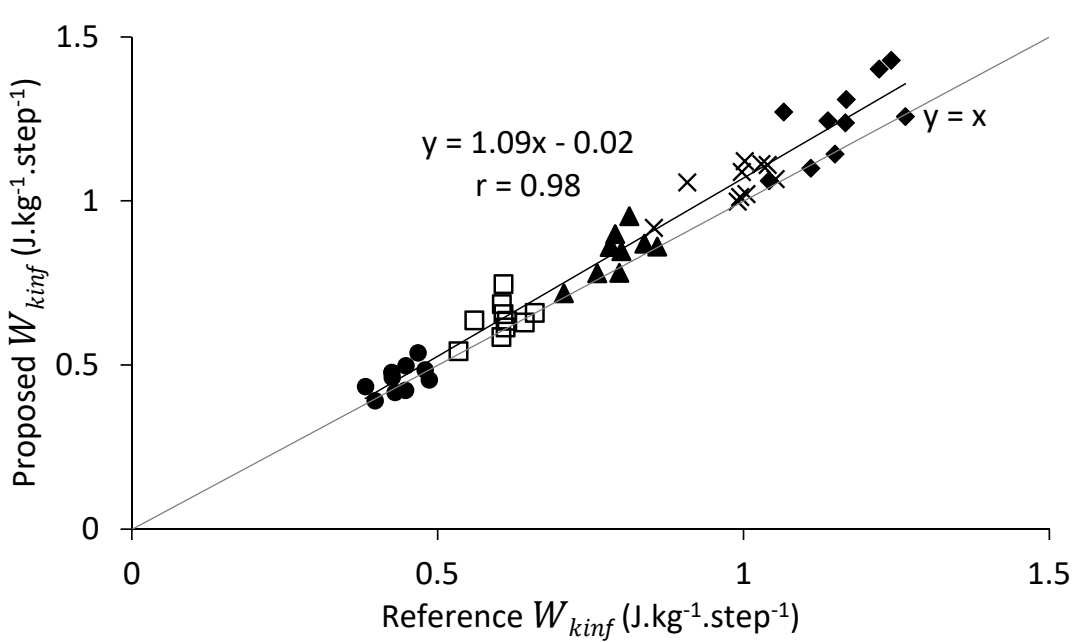


Table 1

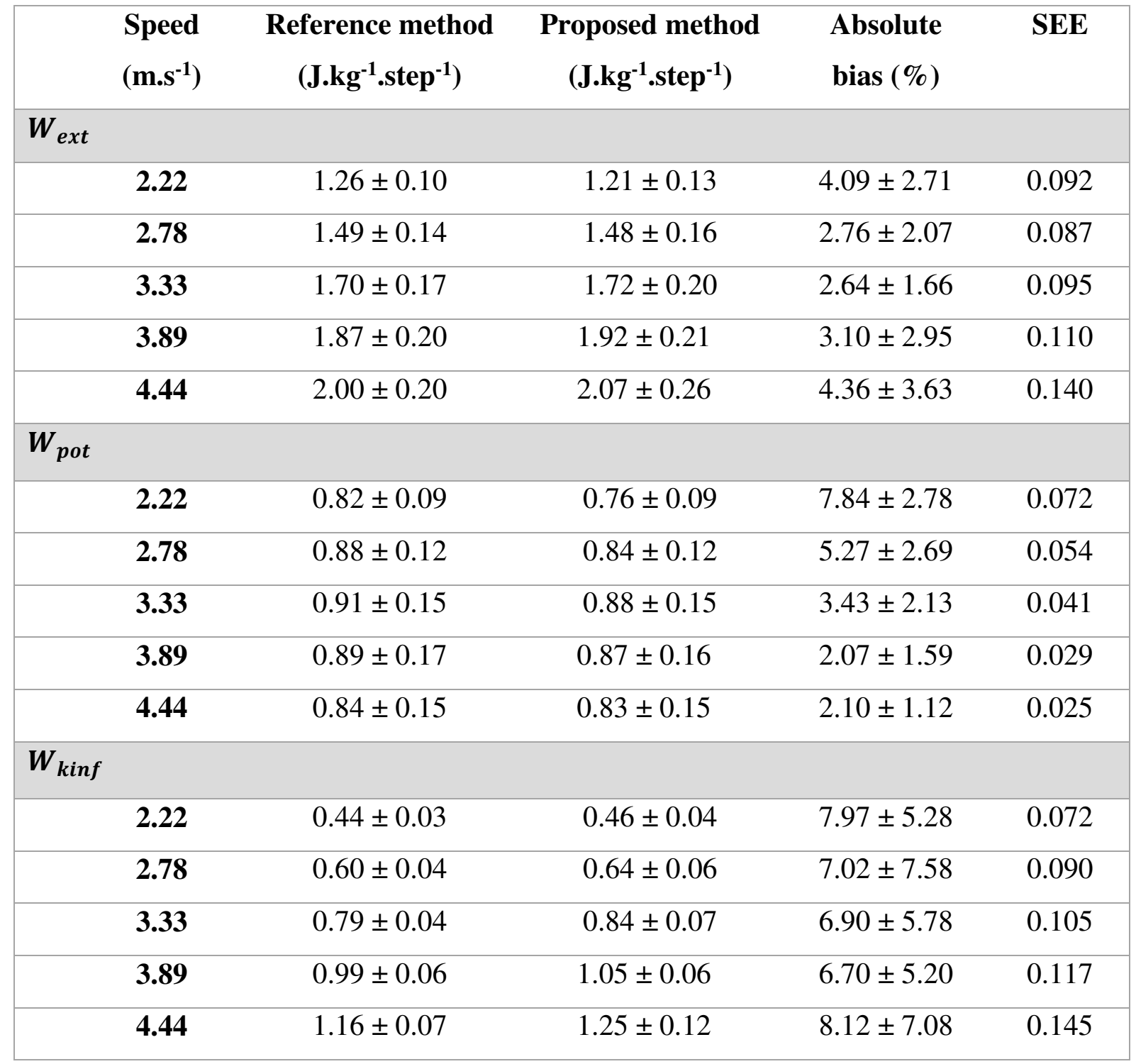

